\title{
Use of Three Dimensional Conformal Radiation Therapy for Node Positive Breast Cancer Does Not Result in Excess Lung and Heart Irradiation
}

\author{
Phillip Prior ${ }^{1}$, Irina Sparks' ${ }^{1}$ J. Frank Wilson'1, Joseph Bovi1, Adam Currey ${ }^{1 *}$, Julie Bradley ${ }^{1}$, \\ Tracy Kelly ${ }^{1}$, X. Allen Li ${ }^{1}$, Julia R White ${ }^{2}$
}

\footnotetext{
${ }^{1}$ Department of Radiation Oncology, Medical College of Wisconsin, Milwaukee, WI, USA

${ }^{2}$ Department of Radiation Oncology, Ohio State University, Columbus, OH, USA

Email: *acurrey@mcw.edu
}

How to cite this paper: Prior, P., Sparks, I., Wilson, J.F., Bovi, J., Currey, A., Bradley, J., Kelly, T., Li, X.A. and White, J.R. (2017) Use of Three Dimensional Conformal Radiation Therapy for Node Positive Breast Cancer Does Not Result in Excess Lung and Heart Irradiation. International Journal of Medical Physics, Clinical Engineering and Radiation Oncology, 6, 1-9.

https://doi.org/10.4236/ijmpcero.2017.61001

Received: November 23, 2016

Accepted: January 8, 2017

Published: January 11, 2017

Copyright $\odot 2017$ by authors and Scientific Research Publishing Inc. This work is licensed under the Creative Commons Attribution International License (CC BY 4.0).

http://creativecommons.org/licenses/by/4.0/

\section{(c) (i) Open Access}

\begin{abstract}
Purpose: This work evaluates the use of target and organs at risk (OAR) dose-volume goals in 3D conformal radiotherapy (3DCRT) planning for node positive breast cancer (NPBC) patients undergoing regional nodal irradiation after lumpectomy/mastectomy. Methods: Dosimetric data for 262 NPBC patients receiving regional nodal and whole breast/chest wall $(\mathrm{WB} / \mathrm{CW})$ irradiation from 2000-2009 were analyzed. In all cases, target \& OAR volumes were delineated on treatment CT scans for field generation and dose-volume histograms (DVHs) were generated. Cases were analyzed to identify how frequently they met treatment planning institutional dose-volume goals ("institutional guidelines" \& standardized in 2005) and how this would affect OAR doses. Results: The incidence of cases from 2000-2009 meeting current institutional guidelines improved over the study period. Target coverage improved from 2005-2009, when guidelines were followed as a part of the plan approval. Those cases from 2000-2004 meeting acceptable target goals were found to be significantly different from those cases from 2005-2009 ( $p<0.01$ ). However, no significant difference between cases meeting OAR goals for plans from 2000-2004 versus 2005-2009 was found. Conclusions: The use of institutional guidelines in 3DCRT for $\mathrm{WB} / \mathrm{CW}$ and regional nodal irradiation for NPBC patients improved target coverage without a statistically significant increase in heart and lung doses.
\end{abstract}

\section{Keywords}

Node Positive Breast Cancer, Dose-Volume Goals, Target Coverage, CT Based Planning 


\section{Introduction}

Historically, breast radiation therapy (RT) treatment planning for node positive disease has involved creating a standard two-dimensional (2DRT) wedged plan on a single transverse contour taken through the center of the breast and then matched to a single anterior field to encompass the supraclavicular-axilla dosed to a depth of $3 \mathrm{~cm}$ [1]. Other disease sites have demonstrated an improvement in the therapeutic ratio using three dimensional conformal RT (3DCRT) or intensity modulated RT (IMRT), where the internal/external anatomical tissues/ organs are delineated and used in treatment planning. Clinical delivery for breast treatment using 2DRT is still common despite two clinical trials demonstrating a reduction in acute and late adverse effects when using 3DCRT/IMRT compared to 2DRT [2] [3]. More recently, contouring atlases for breast have been developed and the use of dose-volume goals has become a standard element of RT planning for patients on more recent Radiation Therapy Oncology (RTOG) and National Surgical Adjuvant Breast and Bowel Project (NSABP) breast clinical trials [4] [5]. However, the uses of 3DCRT/IMRT with target and organs at risk (OAR) dose-volume goals for NPBC patients undergoing regional nodal irradiation after lumpectomy/mastectomy have yet to be standardized nor has the use of such goals gained widespread use in standard practice. We have reviewed our own experience with 3DCRT for node positive breast cancer (NPBC) and have reported excellent local control, survival, and toxicity [6] [7]. The purpose of this work is to retrospectively evaluate the plan quality of NPBC patients since our institution standardized the use of dose-volume goals (referred to as goals hereafter) in treatment planning of NPBC.

\section{Methods}

\subsection{Patient Selection}

A total of 262 NPBC patients were selected for this study and demographically consists of $81.1 \%(\mathrm{n}=212$ cases) Caucasians, 39.4\% $(\mathrm{n}=40)$ African Americans, 7.6\% $(\mathrm{n}=8)$ Asians and 2 from Southeast Asia/India. Median patient age was 50 (27 - 91), 52.35\% were premenopausal, $75.74 \%$ had positive estrogen receptors, $66.27 \%$ had positive progesterone receptors, and $15.92 \%$ were HER-2 positive (3+ Hercept or amplified by FISH). Mean number of all LNs recovered was 17.1 (1 - 46), mean positive LNs: 5 (1 - 29), extra capsular invasion: 47.31\%). Mean microscopic tumor size was $3.73(0.1-21) \mathrm{cm}$. Staging was pII in $43.3 \%$ and pIII in $52 \%$. 52.3\% underwent lumpectomy and $45.93 \%$ mastectomies. $93.0 \%$ had systemic chemotherapy ( $63.4 \%$ adjuvant \& $29.7 \%$ neoadjuvant), with $90.3 \%$ anthracycline based regiment.

Dosimetry data for these patients receiving conventionally fractionated (i.e. 1.8 - 2.0 Gy per fraction) regional nodal and whole breast/chestwall (WB/CW) irradiation from 2000-2009 at our institution were analyzed without any modifications to contoured structures or plan objects [e.g. ports, blocks, aperature etc. Some exceptions were made in order to faithfully reconstruct the dose dis- 
tribution delivered to the patient] and included in our analysis. All patients with biopsy proven breast cancer that spread to regional lymph nodes (i.e. pathologically node positive) who received 3DCRT to breast and regional lymph nodes as part of post-operative treatment were included in our analysis. Patients who received initial 3DCRT for recurrence or metastatic spread of cancer were excluded. The target (including axillary node levels 2 - 3) \& OAR volumes were delineated on free breathing axial CT scans in all cases. During 2000-2004, evaluation of 3D treatment planning was performed by empirically (see discussion) evaluating the dosimetric coverage to contoured structures but plans were approved per individual physician's preference. Treatment planning goals were institutionally standardized for NPBC in 2005 and subsequently used as part of plan approval. These "institutional guidelines" will be used in our analysis of dosimetric target coverage and OAR doses for all plans generated from 2000-2009.

\subsection{Treatment Planning and Contouring}

The target volumes were delineated on all cases and the dose prescribed according to the International Commission on Radiation Units and Measurement (ICRU) Report 50 and 62 recommendations. Over the study period the method of contouring the whole breast changed. In this study, 187 of 262 (71.3\%) of cases were whole breast, post-lumpectomy and were contoured to reflect the clinical breast tissue wired by the physician at CT simulation, which included the lumpectomy cavity. In 2005 and after, the breast excluded the chest musculature (pectoralis and serratus anterior muscles) and $5 \mathrm{~mm}$ from the skin surface on all cases. Typically the breast did not extend laterally past the mid-axillary line or medially past the sternal-costal interface. Seventy-five of 262 (28.7\%) cases were treated after mastectomy. The clinical extent of the chestwall and scar were determined by the treating physician with wires placed at the time of CT simulation. The chestwall target volume included surgical changes visualized by the physician on CT and, around 2005 and after, did not extend deeper than ribs. The supraclavicular and axilla nodal targets (levels 2 - 3) were contoured in every case. The internal mammary lymph node chain (IMC) (defined by the intercostal artery and vein in the first 3 intercostal spaces) was contoured on 210 of $262(80.2 \%)$ of cases; however the IMCs were not targeted in the remaining 52 of $262(19.8 \%)$ cases. The contour of the lung was outlined using an auto-threshold segmentation algorithm. The heart was contoured by delineating both ventricles and the left atria. The heart was contoured mainly for left-sided NPBC patients; however the heart was contoured for some right-sided NPBC patients.

All cases over the study period were reconstructed using XiO treatment planning system [Elekta AB. (Stockholm, Sweden)] without modification to the original structure contours. Dose was calculations were performed using the fast superposition algorithm with inhomogeneity corrections turned on. The dose was prescribed to the ICRU reference point which was located in the lumpectomy or chestwall volume centroids. Each patient's dose-volume histogram (DVH) was used to calculate the acceptable goals listed in Table 1 for the WB, CW, 
Table 1. Manuscript's institutional dose-volume constraints for 3D conformal radiation therapy treatment planning for node positive breast cancer put in effect in 2005.

\begin{tabular}{llcc}
\hline Definitions of Constraints & \multicolumn{1}{c}{ Site/OAR } & Ideal dose-volume constraints & Acceptable dose-volume constraints \\
\hline Targets & Chestwall/whole breast & $D_{95 \%} \geq 47.5 \mathrm{~Gy}$ & $D_{90 \%} \geq 45.0 \mathrm{~Gy}$ \\
& Axillary Lymph Node & $D_{95 \%} \geq 45.6 \mathrm{~Gy}$ & $D_{90 \%} \geq 43.0 \mathrm{~Gy}$ \\
& Supraclavicular Lymph Node & $D_{95 \%} \geq 45.6 \mathrm{~Gy}$ & $D_{90 \%} \geq 43.0 \mathrm{~Gy}$ \\
& Internal Mammary Lymph Nodes & $D_{90 \%} \geq 42.0 \mathrm{~Gy}$ & $D_{90 \%} \geq 38.0 \mathrm{~Gy}$ \\
Organs at Risk (OAR) & Ipsilateral Lung & $V_{20} \leq 25 \%$ & $V_{20} \leq 30 \%$ \\
& Heart & $V_{25} \leq 5 \%$ & $V_{25} \leq 9 \%$ \\
\hline
\end{tabular}

regional lymph node structures, lung and heart. This dose-volume analysis was repeated for all patients in order to assess those cases meeting the acceptable institutional guidelines listed in Table 1.

\subsection{Treatment Planning Goals}

In this study, we sought to compare dose-volume analyses from 3D treatment plans for NPBC cases treated from 2000-2004 versus those from 2005-2009 in order to assess the impact of using institutional guidelines (Table 1). Specifically, we wanted to evaluate how the standardization of treatment planning goals in 2005 to cover $95 \%$ of the target volume by $95 \%$ of the prescription dose (46 - 50 Gy to WB/CW and regional nodes) affected the dose to the OARs. An ideal and acceptable goal was established for each target and OAR (Table 1). Ideal target coverage was defined as $D_{95 \%} \geq 95 \%$ of the prescription dose, where $D_{95 \%}$ is the dose irradiated to $95 \%$ of the volume or more. Acceptable target coverage was defined as $D_{90 \%}>90 \%$ of the prescription dose. For the targets, the ideal goals are $-95 \%$ of the $\mathrm{WB} / \mathrm{CW}$ volume receiving a dose greater than $47.5 \mathrm{~Gy}\left(D_{95 \%} \geq\right.$ $47.5 \mathrm{~Gy}$ ), both the axillary (AX) and supraclavicular (SCL) lymph nodes $-D_{95 \%} \geq$ $45.6 \mathrm{~Gy}$, and IMC lymph nodes $-D_{90 \%} \geq 42 \mathrm{~Gy}$. The ideal goals for the OARs are $25 \%$ of the ipsilateral lung volume receiving $20 \mathrm{~Gy}\left(V_{20} \leq 25 \%\right)$, and the heart, $V_{25} \leq 5 \%$. Acceptable target coverage was defined as a WB/CW- $\mathrm{D}_{90 \%} \geq 45 \mathrm{~Gy}$, $\mathrm{AX}-\mathrm{D}_{90 \%} \geq 43 \mathrm{~Gy}, \mathrm{SCL}-\mathrm{D}_{90 \%} \geq 43 \mathrm{~Gy}$, and IMC $-\mathrm{D}_{90 \%} \geq 38 \mathrm{~Gy}$. The acceptable goals for the OARs were defined as $V_{20} \leq 30 \%$ and $V_{25} \leq 9 \%$ for the lung and heart, respectively. Note: The heart $V_{45}$ and lung $V_{10}$ were retrospectively collected and presented.

\subsection{Analysis and Statistical Methods}

We hypothesized that the use of institutional guidelines in treatment planning would lead to a statistically significant difference in target coverage in cases from 2005-2009 compared to 2000-2004. A patient's plan was regarded as having met target goals if any 3 of the WB/CW, SCL, AX or IMC DVHs met the acceptable institutional guidelines in Table 1 and the patient's plan was scored a value of 1 . If the acceptable institutional guidelines were not met, the plan was scored a value of 0 . All patient plans were analyzed in this way and the number cases 
meeting target goals for each time period was tallied. A two-tailed $t$-test was performed to determine any statistically significant difference in the proportion of cases meeting target goals from 2000-2004 to those cases from 2005-2009.

Furthermore, we hypothesized that meeting planning target goals would not lead to a statistically significant increase in OAR doses. A patient's plan was regarded as meeting OAR goals if both lung and heart DVHs met the acceptable institutional guidelines in Table 1 and the patient's plan was scored a value of 1 . The plan was scored a value of 0 if the acceptable "institutional guidelines were not met. All patient plans were analyzed in this way and the number of cases meeting OAR goals for period was tallied. A two-tailed $t$-test was performed to determine any statistically significant difference in the proportion of cases meeting OAR goals from 2000-2004 to those cases from 2005-2009.

\section{Results}

\subsection{Meeting Target Goals}

The proportion of cases during the entire study period from 2000-2009 meeting the acceptable institutional guidelines was: $72.1 \% \mathrm{WB} / \mathrm{CW}, 83.5 \%$ SCL, $72.0 \%$ $\mathrm{AX}, 71.9 \%$ IMC, $83.2 \%$, lung, and $92.7 \%$ heart. The total number of $\mathrm{WB} / \mathrm{CW}$, AX, SCLV and IMC cases during 2000-2004 and 2005-2009 were: 130, 130, 130, and 100; and 132, 132,132, and 110, respectively. There were a total of 72 left and 58 right sided WB/CW cases, respectively, during 2000-2004 and 66 left and 66 right sided WB/CW cases, respectively, during 2005-2009. A significant increase in the proportion of cases meeting target goals is seen during 2005-2009 compared with 2000-2004, as shown in Table 2, ( $\mathrm{p}<0.01$; 95\% confidence interval (CI) for the difference in means of $[0.376,0.581])$. This improved target coverage is further demonstrated when looking at the increased mean $D_{90 \%}$ for $\mathrm{WB} / \mathrm{CW}, \mathrm{SCL}, \mathrm{AX}$, and IMC cases generated from 2005-2009 compared to 2000-2004 (Table 3).

Table 2. Percentage of cases broken down by year meeting acceptable institutional dosevolume constraints in Table 1.

\begin{tabular}{|c|c|c|c|c|}
\hline \multirow{2}{*}{$\begin{array}{l}\text { Time } \\
\text { span }\end{array}$} & $\mathrm{WB} / \mathrm{CW}$ & SCLV & $\mathrm{AX}$ & IMC \\
\hline & $\begin{array}{c}D_{90 \%} \\
(\mathrm{~Gy}) \geq 45 \mathrm{~Gy}\end{array}$ & $\begin{array}{c}D_{90 \%} \\
(\mathrm{~Gy}) \geq 43 \mathrm{~Gy}\end{array}$ & $\begin{array}{c}D_{90 \%} \\
(\mathrm{~Gy}) \geq 43 \mathrm{~Gy}\end{array}$ & $\begin{array}{c}D_{90 \%} \\
(\mathrm{~Gy}) \geq 38 \mathrm{~Gy}\end{array}$ \\
\hline $2000-2004$ & $58.5 \%$ & $71.5 \%$ & $46.2 \%$ & $62.0 \%$ \\
\hline $2005-2009$ & $85.6 \%$ & $93.9 \%$ & $94.7 \%$ & $80.9 \%$ \\
\hline \multirow{2}{*}{\multicolumn{2}{|c|}{ Time span }} & Lung & \multicolumn{2}{|r|}{ Heart } \\
\hline & & $V_{20} \leq 30 \%$ & \multicolumn{2}{|r|}{$V_{25} \leq 9 \%$} \\
\hline \multicolumn{2}{|c|}{$2000-2004$} & $87.7 \%$ & \multicolumn{2}{|r|}{$89.2 \%$} \\
\hline \multicolumn{2}{|c|}{$2005-2009$} & $78.8 \%$ & \multicolumn{2}{|r|}{$96.2 \%$} \\
\hline
\end{tabular}


Table 3. Mean target and organ at risk (OAR) constraints [mean \pm 1 standard deviation] broken down by time period.

\begin{tabular}{ccccc}
\hline \multirow{2}{*}{ Time span } & WB/CW & \multicolumn{1}{c}{ SCLV } & AX & IMC \\
\cline { 2 - 5 } & $D_{90 \%}(\mathrm{~Gy})$ & $D_{90 \%}(\mathrm{~Gy})$ & $D_{90 \%}(\mathrm{~Gy})$ & $D_{90 \%}(\mathrm{~Gy})$ \\
\hline $2000-2004$ & $44.3 \pm 5.8$ & $42.2 \pm 11.3$ & $38.6 \pm 11.4$ & $33.5 \pm 18.3$ \\
$2005-2009$ & $47.4 \pm 5.5$ & $47.6 \pm 6.8$ & $47.3 \pm 5.0$ & $41.4 \pm 14.4$ \\
\hline \multirow{5}{*}{ Time span } & \multicolumn{3}{c}{ Lung } & \multicolumn{2}{c}{ Heart } \\
\cline { 2 - 6 } & $V_{10}(\%)$ & $V_{20}(\%)$ & $V_{25}(\%)$ & $V_{45}(\%)$ \\
\hline $2000-2004$ & $30.9 \pm 14.4$ & $25.5 \pm 13.5$ & $4.7 \pm 4.7$ & $1.4 \pm 2.3$ \\
$2005-2009$ & $34.3 \pm 7.4$ & $26.0 \pm 5.0$ & $4.4 \pm 3.6$ & $1.0 \pm 1.4$ \\
\hline
\end{tabular}

\subsection{Meeting OAR Doses}

A total of 80 cases during 2000-2004 had heart contours (72 \& 8 cases were left \& right breast, respectively), while a total of 72 cases had heart contours from 2005-2009 (66 \& 6 cases were left \& right breast, respectively). No significant difference was found in the proportion of cases meeting both OAR goals for cases generated from 2000-2004 compared to 2005-2009. Although the proportion of cases from 2005-2009 meeting heart $V_{25}$ increased compared to 2000-2004 (Table 2), this was off-set by a decrease in the proportion of cases meeting lung $V_{20}$ in 2005-2009 compared to 2000-2004. The mean $V_{10} \& V_{20}$ for the lung and $V_{25} \& V_{45}$ for the heart (Table 3) further demonstrate a trade-off between achieved lung and heart doses.

\section{Discussion}

In this study, we sought to investigate how the use of institutionally established dose-volume goals for 3DCRT planning of NPBC patients affected the dose delivered to targets and OAR. We demonstrated that using dose-volume goals does not lead to a statistically significant increase in doses to OAR (i.e. heart and lung), a concern that we had at the beginning of this analysis. Our clinical experience suggests that acceptable levels of normal tissue toxicity and good local control can be achieved with these 3DCRT goals in treating NPBC. For example, acute dermatitis Grade $1 \& 2$ was $83.0 \%$ and $13.4 \%$, respectively; Telangiectasia Grade 1 and 2 - 3 were 9\% and 3.2\%, respectively; and only 1 reported case each of both pneumonitis and pericarditis [6] [7]. Furthermore, we have achieved a local control rate of $94.7 \%$ and regional lymph node control of $99.4 \%$ at a median follow-up of 7 years [6] [7].

Even though recent protocols through the NSABP, Alliance Oncology and RTOG have requirements for 3DCRT planning goals for targets and OARs, there is little published about how this impacts treatment planning. However, other reports have documented that more individualized treatment planning could improve target dose coverage and OAR sparing. Kraisin et al. studied 25 patients who underwent $2 \mathrm{D}$ treatment planning for post-lumpectomy breast RT with 
subsequent reconstruction on a 3D treatment planning system [8]. Dose-volume analysis performed on those reconstructed plans found that $64 \%$ of cases achieved adequate coverage of the breast (defined as $95 \%$ of the target receiving at least $95 \%$ of the prescribed dose). Goodman et al. studied 55 patients who had standard 2D fields for supraclavicular and axillary (level 1 - 3) irradiation that then underwent CT and delineation of the supraclavicular and axillary lymph nodes [9]. Evaluation of the CT defined nodal groups demonstrated a significant variation of the included nodes in the $2 \mathrm{D}$ defined treatment fields. Therefore, they concluded that nodal groups should be delineated on CT prior to field definition in order to achieve the desired target coverage. More recently, investigators have focused on using intensity-modulated RT (IMRT) techniques to achieve improved target coverage, avoidance of OAR, and as a means for correcting for dose in homogeneities [10] [11]. For example, Jones et al. planned 10 cases using fixed beam versus helical Tomotherapy for NPBC. This dosimetric study used similar target coverage to compare the different techniques available on Tomotherapy; however only $V_{20}$ lung goal was used for plan optimization. In comparison to the IMRT/Tomotherapy reports, our study demonstrated in a large number of cases $(n=262)$ that using established does-volume goals to meet target coverage and avoidance of OAR is achievable with 3DCRT. Despite a lack of consensus regarding NPBC dose-volume goals in the literature, our OAR institutional guidelines are within or tighter than the recommendations of the Quantitative Analyses of Normal Tissue Effects in the Clinic (QUANTEC) [12].

\section{Conclusions}

In conclusion, our study is the first to investigate the efficacy of using dosevolume goals in treatment planning for NPBC patients. Any retrospective analysis for breast RT of this type has limitations: 1) The variability introduced in the RT planning process wasn't discussed in the literature until 2007-2009 (near the end of our study period), which makes it difficult to assess the impact of this effect on our results; 2) Contouring databases, like the RTOG breast atlas, were not published until 2009, making it more difficult to assess differences in contouring in our analysis; and 3) The heart was not contoured for all WB/CW cases. Any institution should use dose-volume goals with caution given this variability in defining clinical and planning target volumes. One limitation of our retrospective study is that there was no consistent use of institutional dosevolume goals over the time period studied. Prior to 2005, plans were evaluated qualitatively slice by slice to visually ensure isodose lines covered targets and OAR were adequately spared. The DVHs were reviewed but not used to assess a plan as acceptable for treatment. A second limitation is that these goals were generated by our institutional experience well before there was much evidence in the literature regarding the optimal dose-volume goals for NPBC. In particular, recent breast cancer trails (e.g. NSABP B-51) have different heart goals for right and left sided WB/CW cases but our study used the same goals for both WB/CW cases [5]. However, the strengths of this study are that it is the first to our know- 
ledge to demonstrate in a large number of cases the feasibility of using dosevolume goals on DVHs to achieve an optimal 3DCRT treatment plan for NPBC. In the future, clinical trials and prospective databases evaluating the treatment of node positive breast cancer will be ideally suited for prospective confirmation of these dose-volume goals.

\section{Acknowledgements}

This research was partially supported by Komen Breast Cancer Foundation and by MCW Radiation Oncology Folkman Funds.

\section{References}

[1] White, J., Moughan, J., Pierce, L.J., Morrow, M., Owen, J. and Wilson, J.F. (2004) Status of Postmastectomy Radiotherapy in the United States: A Patterns of Care Study. International Journal of Radiation Oncology ${ }^{*}$ Biology ${ }^{*}$ Physics, 60, 77-85. https://doi.org/10.1016/j.ijrobp.2004.02.035

[2] Pignol, J., Olivotto, I., Rakovitch, E., Gardner, S., Sixel, K., Beckham, W., Vu, T.T.T., Truong, P., Ackerman, I. and Paszat, L. (2008) A Multicenter Randomized trial of Breast Intensity-Modulated Radiation Therapy to Reduce Acute Radiation Dermatitis. Journal of Clinical Oncology, 26, 2085-2092. https://doi.org/10.1200/JCO.2007.15.2488

[3] Donovan, E., Bleakley, N., Denholm, E., Evans, P., Gothard, L., Hanson, J., Peckitt, C., Reise, S., Ross, G., Sharp, G., Symonds-Tayler, R., Tait, D. and Yarnold, J. (2007) Randomised Trial of Standard 2D Radiotherapy (RT) versus Intensity Modulated Radiotherapy (IMRT) in Patients Prescribed Breast Radiotherapy. Radiotherapy and Oncology, 82, 254-264. https://doi.org/10.1016/j.radonc.2006.12.008

[4] Li, X.A., Tai, A., Arthur, D.W., Buchholz, T.A., Macdonald, S., Marks, L.B., Moran, J.M., Pierce, L.J., Rabinovitch, R., Taghian, A., Vicini, F., Woodward, W. and White, J.R. (2009) Variability of Target and Normal Structure Delineation for Breast Cancer Radiotherapy: An RTOG Multi-Institutional and Multiobserver Study. International Journal of Radiation Oncology * Biology * Physics, 73, 944-951.

https://doi.org/10.1016/j.ijrobp.2008.10.034

https://doi.org/10.1158/1538-7445.sabcs15-ot2-02-02

[5] Mamounas, E., Bandos, H., White, J., Julian, T., Khan, A., Shaitelman, S., Torres, M., Vicini, F., Ganz, P., McCloskey, S., Paik, S., Gupta, N., Li, X., DiCostanzo, D., Costantino, J., Curran, W. and Wolmark, N. (2016) Abstract OT2-02-02: NRG Oncology/NSABP B-51/RTOG 1304: A Phase III Clinical Trial to Determine If Chest Wall and Regional Nodal Radiotherapy (CWRNRT) Post Mastectomy (Mx) or the Addition of RNRT to Breast RT Post Breast-Conserving Surgery (BCS) Will Reduce Invasive Cancer Events in Patients (pts) with Positive Axillary (Ax) Nodes Who Are ypN0 after Neoadjuvant Chemotherapy (NC). Cancer Research, 76, OT2-02-02. http://cancerres.aacrjournals.org/content/76/4_Supplement/OT2-02-02

[6] Sparks, I., Prior, P., Xiang, Q., Li, X.A., Yen, T.W. and White, J.R. (2011) Lymphedema and Arm Symptoms Following Three-Dimensional Conformal Radiation Therapy (3DCRT) for Node-Positive Breast Cancer (BC) Patients. Journal of Clinical Oncology, 29, 130.

http://ascopubs.org/doi/abs/10.1200/jco.2011.29.27_suppl.130

[7] Bradley, J., Prior, P., Sparks, I., Xiang, Q., Santana-Davila, R., Walker, A., Wilson, J., Li, X. and White, J. (2012) P3-13-05: Analysis of Heart Dose-Volume Parameters and Cardiac Events among Node Positive Breast Cancer (NPBC) Patients Treated 
with Three-Dimensional Conformal Radiation Therapy (3D-CRT). Cancer Research, 71, P3-13-05.

http://cancerres.aacrjournals.org/content/71/24_Supplement/P3-13-05

[8] Krasin, M., McCall, A., King, S., Olson, M. and Emami, B. (2000) Evaluation of a Standard Breast Tangent Technique: A Dose-Volume Analysis of Tangential Irradiation Using Three-Dimensional Tools. International Journal of Radiation Oncology ${ }^{*}$ Biology ${ }^{*}$ Physics, 47, 327-333.

https://www.ncbi.nlm.nih.gov/pubmed/10802356

https://doi.org/10.1016/S0360-3016(00)00449-1

[9] Goodman, R.L., Grann, A., Saracco, P. and Needham, M.F. (2001) The Relationship between Radiation Fields and Regional Lymph Nodes in Carcinoma of the Breast. International Journal of Radiation Oncology ${ }^{\star}$ Biology ${ }^{\star}$ Physics, 50, 99-105.

https://www.ncbi.nlm.nih.gov/pubmed/11316551 https://doi.org/10.1016/S0360-3016(00)01581-9

[10] Barnett, G.C., Wilkinson, J., Moody, A.M., Wilson, C.B., Sharma, R., Klager, S., Hoole, A.C.F., Twyman, N., Burnet, N.G. and Coles, C.E. (2009) A Randomised Controlled Trial of Forward-Planned Radiotherapy (IMRT) for Early Breast Cancer: Baseline Characteristics and Dosimetry Results. Radiotherapy and Oncology, 92, 34-41. https://doi.org/10.1016/j.radonc.2009.03.003

[11] Barnett, G.C., Wilkinson, J.S., Moody, A.M., Wilson, C.B., Twyman, N., Wishart, G.C., Burnet, N.G. and Coles, C.E. (2012) Randomized Controlled Trial of ForwardPlanned Intensity Modulated Radiotherapy for Early Breast Cancer: Interim Results at 2 Years. International Journal of Radiation Oncology ${ }^{\star}$ Biology ${ }^{\star}$ Physics, 82, 715 723. https://doi.org/10.1016/j.ijrobp.2010.10.068

[12] Gagliardi, G., Constine, L.S., Moiseenko, V., Correa, C., Pierce, L.J., Allen, A.M. and Marks, L.B. (2010) Radiation Dose-Volume Effects in the Heart. International Journal of Radiation Oncology ${ }^{*}$ Biology ${ }^{*}$ Physics, 76, S77-S85.

https://doi.org/10.1016/j.ijrobp.2009.04.093

Submit or recommend next manuscript to SCIRP and we will provide best service for you:

Accepting pre-submission inquiries through Email, Facebook, LinkedIn, Twitter, etc. A wide selection of journals (inclusive of 9 subjects, more than 200 journals)

Providing 24-hour high-quality service

User-friendly online submission system

Fair and swift peer-review system

Efficient typesetting and proofreading procedure

Display of the result of downloads and visits, as well as the number of cited articles

Maximum dissemination of your research work

Submit your manuscript at: http://papersubmission.scirp.org/

Or contact ijmpcero@scirp.org 